\title{
Diffusion processes: invertibility problem and guaranteed estimation theory
}

\author{
Irina F.Sivergina * \\ Institute for Mathematics and Mechanics, Urals Branch, RAS \\ 16 S.Kovalevskoj Str., 620219, Ekaterinburg, Russia. \\ Tel.: 3432-493243. Fax: 3432-442581. E-mail: siv@oou.imm.intec.ru
}

\begin{abstract}
This paper considers a series of problems on state and parameter specifying for distributed parameter systems on the basis of measurements generated by sensors. Observability and invertibility issues, duality relations for problems of control, schemes of sensor's generating to guarantee the observability desired are discussed. These problems are treated within a unified framework of guaranteed estimation procedures for systems with unknown but bounded errors.
\end{abstract}

\section{Keywords}

Inverse problem, distributed parameter system, sensor, invertibility, observability, duality, guaranteed estimation problem, regularization

\section{INTRODUCTION}

This paper deals with the problem of parameter and state specifying for distributed parameter system on the basis of measurements generated by sensors. The issues treated here are the invertibility and observability problems. The approach leans upon describing the system properties in terms of solutions to some guaranteed estimation problems. They are formulated for the systems with disturbances taken to be unknown but bounded. That provides another interpretation for the properties known and reveals quite new features. The necessary and sufficient conditions to provide them are given. The duality relations for the problems of control are considered.

Another aspect of the invertibility problem considered here is in generating sensors ensuring a desired invertibility property for an evolutionary system. A procedure how to do this for the strong observability is discussed. It makes use of results on optimization of observers for guaranteed estimation problem. Regulating the parameters in that problem enables a sensor with the desired property to be approximated.

\footnotetext{
${ }^{*}$ Work supported by the Russian Fund of Fundamental Researches under Projects 94-01-00803 and 95$01-00380$ 


\section{BASIC NOTIONS}

Let there be the initial-boundary problem

$$
\begin{cases}\frac{\partial u(t, x)}{\partial t}=\Delta u(t, x)+f(t, x), & t \in(0, T), x \in \Omega \\ u(0, x)=u_{0}(x), & x \in \Omega ; \\ u(t, \zeta)=v(t, \zeta), & t \in(0, T), \zeta \in \partial \Omega\end{cases}
$$

Here $\Omega$ is an open bounded domain in an n-dimensional space having a piecewise-smooth boundary $\partial \Omega$. A symmetric uniformly elliptic operator with the smooth coefficients may stay instead of Laplacian $\Delta$. Here $f(t, x)$ is the control function, $v(t, x)$ is the boundary condition, $u_{0}(x)$ is the initial distribution. They are supposed to be square integrable functions on the proper sets. We'll consider $u(t, x)$ as a generalized solution to (1).

There is an evolutionary system having been associated with that initial-boundary problem. The state of the system at a time instant $t, 0 \leq t \leq T$, is the function $u(t, \cdot)$ being an element of the space $L_{2}(\Omega)$. The triple $w=\left(f(\cdot, \cdot), v(\cdot, \cdot), u_{0}(\cdot)\right)$ will be referred to as an input for that system.

Further it is assumed that the input is taken to be unknown in advance. It is supposed that all the available information on the solution is given through a finite-dimensional measurement equation

$y(t)=G(t) u(t, \cdot), \quad 0 \leq t \leq T$.

$y(t)$ is a measurement data, $y(t) \in R^{m}, y(\cdot) \in L_{2}^{m}(0, T)$. $G(t)$ is a linear observation operator, or sensor. Let us start with two traditional notions.

Definition 1 We will say that the system (1),(2) is invertible if the measurement $y(t) \equiv$ $0,0 \leq t \leq T$, yields $w=0$.

Consider the initial boundary problem (1) assuming that

$f(t, x) \equiv 0, \quad v(t, x) \equiv 0$.

Definition 2 We will say that the system (1)-(3) is observable if the measurement $y(t) \equiv$ $0,0 \leq t \leq T$, yields $u(T, \cdot)=0$.

The first definition is equivalent to the fact that the linear mapping $C, C w=y(\cdot)$ is such that $\operatorname{Ker} C=\{0\}$. So, if the system is invertible then two different inputs yield two different measurements. The second one implies the similar property for the mapping $D u(T, \cdot)=y(\cdot)$.

There are papers studying these properties and sensors to assure them for the system. Let us think over these properties from another point of view. From here on we are concentrating on the observability problem. We'll return to the invertibility one later. 
Let us consider a guaranteed estimation problem closely related to the observability one. Suppose, that the measurement $y(t)$ is not exact and contains additively an uncertain disturbance $\xi(t)$

$$
\begin{aligned}
& y(t)=G(t) u(t, \cdot)+\xi(t), \quad 0 \leq t \leq T, \\
& \|\xi(\cdot)\|_{L_{2}^{m}(0, T) \leq \mu, \quad \mu \geq 0 .}
\end{aligned}
$$

That inequality gives an a priori restriction on $\xi(t)$. Now, in general, there's no way of determining the state $u(T, \cdot)$ of the system on the basis of the measurement data $y(t)$. This inverse problem, as a rule, has got a non-unique solution. This leads us to the following notion (Kurzhanski, 1977).

Definition 3 The informational set $\mathcal{U}[y]$ of states $u(T, \cdot)$ of the system (1), (3)-(5) being consistent with measurement data $y(t)$ and the a priori restriction on $\xi(t)$, is the set of all those functions $z(\cdot) \in L_{2}(\Omega)$ for each of which there exists an input $w$ such that the corresponding solution $u(t, \cdot)$ to the problem (1) at the time instant $T$ will be equal to $z(\cdot)$ and the equation (4) will be valid for some $\xi(t)$ satisfying the a priori restriction (5).

The linearity of the system and the convexity of the set described by (5) imply the convexity of $\mathcal{U}[y]$. This set is said to be a solution to the guaranteed estimation problem of the state $u(T, \cdot)$ on the basis of $y(\cdot)$. We can state the first obvious statement.

Lemma 1 The system (1),(3) is observable if and only if $\mathcal{U}[y]$ is one-point set for $\mu=0$, whatever is $y(t)$.

If the informational set was bounded, one might be defined the minimax estimate of the state $u(T, \cdot)$, being the Chebyshev centre of $\operatorname{cl} \mathcal{U}[y]$. It is determined through the formula $u^{0}=\arg \min _{u \in L_{2}(\Omega)} \sup _{v \in \mathcal{U}[y]}\|u-v\|_{L_{2}(\Omega)}$. (A solution to that variational problem does exist and is unique under the assumption above.) If our system were finite dimensional, i.e. were described by a system of ordinary differential equations, the observability would guarantee the existence of the minimax estimate $u^{0}$. Our infinite dimensional system needs a more strong property to ensure that. Namely,

Definition 4 (Kurzhanski, Khapalov, 1991) We will say that the system (1),(3)-(5) is strongly observable if $\mathcal{U}[y]$ is bounded, whatever is the measurement $y(t)$.

This definition is equivalent to the continuous observability of the system implying the existences of a constant $\alpha>0$ such that the inequality $\|u(T, \cdot)\|<\alpha\|y\|$ is true, whatever is $y=G(t) u(t, \cdot), 0 \leq t \leq T$. That means that the operator $D$ is continuously invertible. It is clear that Definition 4 implies Definition 2. It turns out that there are other substantial properties "between" them.

\section{$3 \epsilon$-OBSERVABILITY}

A set in a Hilbert space is bounded if and only if the projections of it onto all the onedimensional subspaces are bounded. So, the system (1),(3)-(5) is just strongly observable 
if and only if the projections of $U[y]$ onto all the one-dimensional subspaces are bounded, whatever is $y(t)$. It should be emphasized that if the boundedness were inherent in the intersections of the informational set with all the one-dimensional subspaces, whatever the measurement is, the system would be observable. Let us now give the following

Definition 5 (Kurzhanski, Sivergina, 1991) We will say that the system (1),(3)-(5) is $\epsilon$-observable if for almost every functions $\varphi \in L_{2}(\Omega)$ the projection of $\mathcal{U}[y]$ onto a subspace generated by that is bounded, whatever is the measurement $y(t)$.

Theorem 1 The system (1),(3)-(5) is $\epsilon$-observable if and only if $D$ is invertible and has a closed extension.

Theorem 2 Let for almost every functions from $L_{2}(\Omega)$ the projection of $\mathcal{U}[0]$ onto the subspace generated by that be bounded, whatever $y(t)$ is. Then the system (1),(3)-(5) is $\epsilon-$ observable.

So, we have three notions related to the observability issue: the observability, the $\epsilon$ observability, and the strong observability. It has to be shown that they are distinct.

Example 1 That is an instance of a system being observable but not $\epsilon$ - observable. Suppose the sensor is of spatially averaged type:

$G(t) u(t, \cdot)=\int_{\Omega} g(t, x) u(t, x) d x, 0 \leq t \leq T$

where $g(t, x)=\varphi_{1}(x) e^{\lambda_{1}(t-T)} e^{-t}+\sum_{k=2}^{\infty} \varphi_{k}(x) e^{\lambda_{k}(t-T)}\left(e^{-k^{2} t}-\frac{k}{k-1} e^{-k-1)^{2} t}\right) \cdot \varphi_{i}(\cdot), \lambda_{i}$ are the eigen-functions and the eigen-values for the spectral problem $\Delta \varphi_{i}(x)=-\lambda_{i} \varphi_{i}(x), x \in$ $\Omega, \quad \varphi_{i}(\zeta)=0, \zeta \in \partial \Omega ; \lambda_{i} \rightarrow \infty$ as $i \rightarrow \infty$. $\left\{\varphi_{i}\right\}_{i=1}^{\infty}$ make up an orthonormal base in $L_{2}(\Omega)$. It is easy to show that the condition $G(t) u(t, \cdot) \equiv 0$, implies $u(T, \cdot)=0$. So, the system is observable. At the same time, the set $\mathcal{U}[0]$ is unbounded. Moreover, a closure of it contains a whole linear subspace generated by the function

$\varphi(x)=\sum_{i=1}^{\infty} \frac{1}{i} \varphi_{i}(x), \quad x \in \Omega$

Note, in that Example there is no restriction on $n$, i.e. on the dimension of the space variable. It might be anyone.

Example 2 The instance of a system being $\epsilon$-observable but not strongly observable gives the heat equation in the domain $\Omega=(0,1)$ with the spatially averaged sensor $(6)$ when $g(t, x)$ doesn't depend on $t$ and is equal to $\varphi(x)$ from (7). Now, because of the minimality property in $L_{2}(\Omega)$ of the system $\left\{e^{-\lambda_{i} t}, \quad 0 \leq t \leq T, i=1,2, \ldots\right\}$ we have got $\left|u_{0 i}\right| \leq \alpha_{i}(\|y(\cdot)\|+\mu), \quad i=1,2, \ldots$ where $\alpha_{i}$ doesn't depend on $y(\cdot)$. This assures the $\epsilon$-observability for the system. But, according to Dolecki's result (1977), the system won't be strongly observable because of the equality $\sum_{i=1}^{\infty} \frac{i^{2}}{e^{-\lambda_{i} T}}=\infty$. 


\section{DUAL PROBLEM}

One way of studying the observability property of a system is to turn to the dual control problem. Let us formulate a property being dual to the $\epsilon$-observability. Consider the system

$$
\begin{cases}\frac{\partial v(t, x)}{\partial t}=-\Delta v(t, x)+G^{*}(t) \lambda(t), & t \in(0, T), x \in \Omega \\ v(T, x)=0, & x \in \Omega ; \\ u(t, \zeta)=0, & t \in(0, T), \zeta \in \partial \Omega\end{cases}
$$

Here $\lambda(\cdot) \in L_{2}^{m}(0, T)$ is a control. The control problem consists in selecting the control to bring the system to the time instant 0 to the position $S^{*}(T) \varphi(\cdot)$ :

$u(0, \cdot)=S^{*}(T) \varphi(\cdot)$

$\varphi(\cdot) \in L_{2}(\Omega)$ is a given function, $S(t), t \geq 0$ is the semi-group corresponding to the evolutionary system (1). So, the set of those $\varphi(\cdot)$ for which the problem (8),(9) may be solvable is of interest. Suppose, $G(t)$ in (8) is the same as in (2). It is known, that if the system (1),(3)-(5) is strongly observable, the control problem is solvable for any $\varphi(\cdot)$. If the system is only observable, one is solvable for almost every $\varphi(\cdot) \in L_{2}(\Omega)$. In this case one says about approximate controllability of the system $(8),(9)$ :

$\forall \varphi(\cdot) \in L_{2}(\Omega) \forall \epsilon>0 \exists \lambda(* \cdot): u(0, \cdot) \in S^{*}(T) \varphi+\mathcal{O}_{\varepsilon}(0)$

Theorem 3 The property of the system (1),(3)-(5) to be $\epsilon$-observable is equivalent to the property of the problem (8),(9) to be solvable in the following sense:

$\forall \varphi(\cdot) \forall \epsilon>0 \exists \lambda(\cdot): u(0, \cdot) \in S^{*}(T)\left(\varphi+\mathcal{O}_{\epsilon}(0)\right)$.

It is easy to note that the key to the approach to the observability problem lies with the property of the system to be observable in a direction. We are illustrating that notion with the invertibility one. But before proceeding to that we'll consider another problem connected with the observability problem.

\section{REGULARIZATION OF SENSORS}

Consider the system

$$
\begin{cases}\frac{\partial u(t, x)}{\partial t}=\frac{\partial^{2} u(t, x)}{\partial x^{2}}, & t \in(0, T), x \in \Omega=(0,1) \\ u(t, \zeta)=0, & t \in(0, T), \zeta \in \partial \Omega \\ y(t)=\int_{\Omega} g(x) u(t, x) d x, & 0 \leq t \leq T\end{cases}
$$

Let us suppose a possibility for a function $g(x)$ to be chosen. It is well known that there exist the functions $g(\cdot) \in L_{2}(\Omega)$ for which the system (10) would be strongly observable. 
Moreover they would exist to belong to the set $\mathcal{G}=\left\{g(\cdot) \in C^{1}(\bar{\Omega}) \mid\|g(\cdot)\|_{C^{1}(\Omega)} \leq 1\right\}$. How extract them? The method to do this is proposed here.

Consider an auxiliary observation system, in which the initial distribution $u_{0}$ is unknown, the disturbance $\xi(t)$ is present in the observation equation, and $g(x)$ is a function form $\mathcal{G}$. One supposes $u_{0}$ and $\xi(t)$ to be a priori restricted by the joint quadratic inequality

$$
\begin{cases}\frac{\partial u(t, x)}{\partial t}=\frac{\partial^{2} u(t, x)}{\partial x^{2}}, & t \in(0, T), x \in \Omega, \Omega=(0,1) \\ u(t, \zeta)=0, & t \in(0, T), \zeta \in \partial \Omega \\ y(t)=\int_{\Omega} g(x) u(t, x) d x+\xi(t), & 0 \leq t \leq T \\ \epsilon\left\|u_{0}\right\|_{L_{2}(\Omega)}^{2}+\|\xi(\cdot)\|_{L_{2}(0, T)}^{2} \leq \mu^{2} . & \end{cases}
$$

Here $\epsilon>0$ is a parameter. Formally one defines an informational set $\mathcal{U}_{\epsilon}[y]$ of the states $u(T, \cdot)$ of the system (11) being consistent with the measurement data $y(t)$ and the a priori restriction on $u_{0}$ and $\xi(t)$. It is a closed bounded set in $L_{2}(\Omega)$. We would associate the number $\mathcal{J}_{\epsilon}(g)$ with a sensor in (11) corresponding to a function $g(\cdot) \in \mathcal{G}$

$$
\mathcal{J}_{\epsilon}(g)=\max _{y(\cdot)} \sup _{\|\varphi\| \leq 1} \max _{u(\cdot) \in \mathcal{U}_{\epsilon}[y]}|<\varphi, u>| .
$$

By $\langle\cdot, \cdot\rangle$ it is denoted a scalar production in $L_{2}(\Omega) . \mathcal{J}_{\epsilon}(g)$ may be interpreted as a functional on $\mathcal{G}$. One can show that the external maximum in (12) is reached on the function $y(\cdot)=0$. By applying the methods of paper (Kurzhanski, Khapalov, 1986) we make sure that the variational problem $\min _{g(\cdot) \in \mathcal{G}} \mathcal{J}_{\epsilon}(g)$ is solvable. Denote its solution by $g_{\epsilon}(\cdot)$. There exists a subsequence $g_{\epsilon_{i}}(\cdot)$ being convergent in uniform metric when $\epsilon_{i}$ tends to zero. Let $g_{0}(\cdot)$ be a limit.

Theorem 4 For $g(\cdot)=g_{0}(\cdot)$ the system (10) is strongly observable.

Remark 1 A similar result is true for the point-wise sensors $G(t) u(t, \cdot)=u(t, x(t)), 0<$ $\delta \leq t \leq T$. Here $x(t)$ is a function taking the values in $\bar{\Omega}$. In that case one has to take $\mathcal{G}=\left\{x(\cdot) \in C^{1}(\delta, T)|x(t) \in \bar{\Omega},| \dot{x}(t) \mid \leq 1\right\}$.

\section{INVERTIBILITY PROBLEM}

Now we turn to the invertibility problem. Let us remind the system under consideration. It is (1)-(2). Restrict ourselves to an assumption that the input as well as the sensor have the special representations

$$
f(t, x)=\sum_{i=1}^{s} b_{i}(x) f_{i}(t), v(t, \zeta)=\sum_{i=1}^{k} d_{i}(\zeta) v_{i}(t), G(t) u(t, \cdot)=\operatorname{col}\left[\int_{\Omega} g_{i}(x) u(t, x) d x\right]_{i=1}^{m} .
$$

The functions $b_{i}(x)$ and $d_{i}(x)$ are supposed to be given. So, the invertibility problem is to specify $f_{i}(\cdot), i=1, \ldots, s, v_{i}(\cdot), i=1, \ldots, k$, and $u_{0}(x)$ on the basis of the measurement data $y(t), 0 \leq t \leq T$. Here it is rationally to call the element $\tilde{w}=\left(f_{1}, \ldots, f_{s}, v_{1}, \ldots, v_{k}, u_{0}\right)$ $\in L_{2}^{s}(0, T) \times L_{2}^{k}(0, T) \times L_{2}(\Omega)$ as the input for the system. The following assumptions are 
supposed to be valid from now on: each of the function systems $\left\{b_{i}(\cdot)\right\},\left\{d_{i}(\cdot)\right\}$, and $\left\{g_{i}(\cdot)\right\}$ are linearly independent, and the functions $\left\{b_{i}(\cdot), \sum_{l=1}^{\infty} \int_{\partial \Omega} \frac{\partial \varphi_{l}(\zeta)}{\partial n} d_{j}(\zeta) d \zeta \varphi_{l}(\cdot)\right\}$ are, too.

Formulate some results relative to the invertibility properties of the system. They are presented in (Sivergina, 1995) for a special case.

Theorem 5 For any functions $\left\{b_{i}(\cdot)\right\}_{i=1}^{s},\left\{d_{i}(\cdot)\right\}_{i=1}^{k}$, and for $m=s+k+1$ there exist $g_{1}, g_{2}, \ldots, g_{m}$ such ones that the system will be invertible.

That estimate for $m$ can't be improved. Really, any system being a finite-dimensional Fourier approximation for the observation system under consideration may be invertible only if $m \geq s+k+1$. In the following theorem there are formulated the necessary and sufficient conditions for the system to be invertible in the case that the functions $\left\{b_{i}(\cdot)\right\},\left\{d_{i}(\cdot)\right\}$, and $\left\{g_{i}(\cdot)\right\}$ belong to the space $\stackrel{0}{C}^{\infty}(\Omega)$. Here the techniques of conditionally invariant spaces developed by Basile, Marro (1992) is useful. Denote $G \equiv G(t)$ and define the two operators $\bar{B}$ and $\bar{V}$ mapping from $R^{s}$ and $R^{k}$, respectively, into $L_{2}(\Omega)$ : $\bar{B}\left(f_{1}, \ldots, f_{s}\right)=\sum_{i=1}^{s} b_{i}(x) f_{i}, \bar{V}\left(v_{1}, \ldots, v_{k}\right)=\sum_{i=1}^{k} \sum_{j=1}^{\infty} \int_{\partial \Omega} \frac{\partial \varphi_{i}}{\partial \zeta} d_{i}(\zeta) d \zeta \varphi_{j}(x) v_{i}$.

Theorem 6 The system is invertible if and only if $\bigcup_{l=0}^{\infty} \mathcal{Y}_{l}=L_{2}(\Omega)$, where $\mathcal{Y}_{0}=\operatorname{Im}\left(G^{*}\right)$, $\mathcal{Y}_{l}=\mathcal{Y}_{0}+\Delta\left(\bar{B}^{*}+\bar{V}^{*}\right) \cap \mathcal{Y}_{l-1}$.

\section{INVERTIBILITY IN A DIRECTION}

Now, following the scheme above, consider an information set of inputs, consistent with the measurement data and the restriction on the "noise" in the measurement equation.

Definition 6 The information set $\tilde{\mathcal{W}}[y]$ of inputs $\tilde{w}$ of the system (1),(4),(5) being consistent with the measurement data $y(t)$ and the a priori restriction on $\xi$, is the set of all those inputs $\tilde{w}$ for each of which there exists an element $\xi(\cdot) \in L_{2}^{m}(0, T)$ satisfying the conditions (4),(5).

It is obviously that the strong invertibility property fails. Here the matter of current interest is whether the system is invertible in a direction and if this property is strong. Formulate these notions accurately. Let $\Lambda$ be an element of the input space.

Definition 7 We will say that the system (1),(2) is invertible in a direction $\Lambda$ if $y(t) \equiv 0$ implies that the projection of the input on the subspace generated by $\Lambda$ is equal to 0.

Definition 8 We will say that the system (1),(4),(5) is strongly invertible in a direction $\Lambda$ if the projection of $\tilde{\mathcal{W}}[y]$ on the subspace generated by $\Lambda$ is bounded, whatever $y(\cdot)$ is.

Here it turns it is possible to describe the set of all the directions, in which the system is invertible.

Theorem 7 The system (1),(2) is invertible in the direction $\Lambda$ if and only if $\Lambda$ belongs to a linear space generated by the elements $\Lambda_{t}^{(j)}=\left(f_{1 t}^{(j)}, \ldots, f_{s t}^{(j)}, v_{1 t}^{(j)}, \ldots, v_{k t}^{(j)}, u_{0 t}^{(j)}\right), j=$ 
$1, \ldots, m, 0 \leq t \leq T$ of the form: $u_{0 t}^{(j)}(\cdot)=\sum_{l=1}^{\infty} e^{-\lambda_{l} t} g_{j l} \varphi_{l}(\cdot)$,

$f_{i t}^{(j)}(\tau)=\left\{\begin{array}{ll}\sum_{l=1}^{\infty} e^{-\lambda_{l}(t-\tau)} g_{j l} b_{i l}, & 0 \leq \tau \leq t \\ 0, & t<\tau \leq T\end{array}, v_{i t}^{(j)}(\tau)=\left\{\begin{array}{ll}\sum_{l=1}^{\infty} e^{-\lambda_{l}(t-\tau)} g_{j l} d_{i l}, & 0 \leq \tau \leq t \\ 0, & t<\tau \leq T\end{array}\right.\right.$.

$g_{j l}=\int_{\Omega} g_{j}(z) \varphi_{l}(z) d z, \quad b_{j l}=\int_{\Omega} b_{j}(z) \varphi_{l}(z) d z, \quad d_{j l}=\int_{\partial \Omega} \frac{\partial \varphi_{l}(\zeta)}{\partial n} d_{j}(\zeta) d \zeta$.

To conclude, we formulate the conditions guaranteeing the strong invertibility in a direction for the simplest case.

Theorem 8 Let $s=k=1, m=1, y(\cdot) \in C[0, T]$, the functions $b_{1}(\cdot), d_{1}(\cdot), g_{1}(\cdot)$ be twice continuously differentiable, and the system be invertible in the direction $\Lambda$. Then it is strongly invertible in that direction.

The author is grateful to Academician A.B.Kurzhanski for helpful discussions and encouragement.

\section{REFERENCES}

Basile, F. and Marro, G. (1992) Controlled and conditioned invariants in linear system theory. Prentice-Hall Inc., Englewood Cliffs.

Dolecki, S. (1977) Observability for regular processes. Journal of Mathematical Analysis and Applications, 58, 178-188.

Kurzhanski, A.B.(1977) Control and observation under uncertainty. Nauka, Moscow.

Kurzhanski, A.B. and Khapalov, A.Yu. (1986) On the state estimation problem for distributed systems. Lecture Notes in Control and Information Sciences, 83, 102-113.

Kurzhanski, A.B. and Khapalov, A.Yu. (1991) An observation theory for distributed parameter systems. Journal of Mathematical Systems, Estimation, and Control, 1, 389440.

Kurzhanskii, A.B. and Sivergina I.F. (1992) $\epsilon$-observability for distributed parameter systems. Proceedings of Institute for Mathematics and Mechanics, Urals Branch, RAS. 1, 1222-137.

Sivergina, I.F. (1995) The invertibility and observability problems for evolutionary systems. Doklady of Russian Academy of Sciences. (to appear). 